\title{
Splicing modulation therapy in the treatment of genetic diseases
}

This article was published in the following Dove Press journal:

The Application of Clinical Genetics

4 December 2014

Number of times this article has been viewed

Virginia Arechavala-

Gomeza'

Bernard Khoo ${ }^{2}$

Annemieke Aartsma-Rus ${ }^{3}$

'Neuromuscular Disorders Group, BioCruces Health Research

Institute, Barakaldo, Bizkaia, Spain;

${ }^{2}$ Endocrinology, Division of Medicine,

University College London, London,

UK; ${ }^{3}$ Department of Human Genetics,

Leiden University Medical Center,

Leiden, the Netherlands

All authors contributed equally to this manuscript

Correspondence: Annemieke AartsmaRus

LUMC postzone S4-P, Albinusdreef 2,

2333 ZA Leiden, the Netherlands

Tel +3 I 7I 5269436

Fax +3I 7| 5268285

Email a.m.rus@lumc.nl
Abstract: Antisense-mediated splicing modulation is a tool that can be exploited in several ways to provide a potential therapy for rare genetic diseases. This approach is currently being tested in clinical trials for Duchenne muscular dystrophy and spinal muscular atrophy. The present review outlines the versatility of the approach to correct cryptic splicing, modulate alternative splicing, restore the open reading frame, and induce protein knockdown, providing examples of each. Finally, we outline a possible path forward toward the clinical application of this approach for a wide variety of inherited rare diseases.

Keywords: splicing, therapy, antisense oligonucleotides, cryptic splicing, alternative splicing

\section{Introduction}

Genetic diseases are generally rare diseases caused by mutations in specific genes. Sometimes mutations in different genes can give rise to similar phenotypes, eg, there have been dozens of genes identified in patients suffering from muscular dystrophies. ${ }^{1}$ However, mutations in a single gene can also give rise to multiple diseases with varying phenotypes. Probably the most notorious example is the LMNA gene, in which mutations are associated with multiple phenotypes, including Emery-Dreifuss muscular dystrophy, familial partial lipodystrophy, limb girdle muscular dystrophy, dilated cardiomyopathy, Charcot-Marie-Tooth disease, restrictive dermopathy, and Hutchinson-Gilford progeria syndrome (HGPS). ${ }^{2}$ For a significant number of patients with, or suspected of having a genetic disease, the mutation in the causative gene has not yet been identified. However, with the rapid advances made by "next generation sequencing", it is anticipated that mutations will soon be identified for almost all patients with genetic disorders; the International Rare Disease Research Consortium (IRDiRC) has set itself an ambitious goal of having the means available to diagnose most rare genetic diseases by 2020 (http://www.irdirc.org).

With the availability of next generation sequencing, it is now possible to perform a more in-depth analysis of candidate genes. In the past, generally only exons and the donor and acceptor splice sites (ie, the first and last two base pairs of an intron, respectively) were analyzed. However, it is now feasible to analyze complete introns and there are multiple publications that report deep intronic mutations that activate cryptic splice sites and thus disrupt normal transcript processing. ${ }^{3}$ In parallel, antisense-mediated splicing modulation has been developed from preclinical cell and animal models into the clinical trial phase for Duchenne muscular dystrophy (DMD) and spinal muscular atrophy (SMA). ${ }^{48}$ This approach makes use of antisense oligonucleotides (ASOs, small 
pieces of chemically modified DNA or RNA) and would offer a potential treatment for genetic disorders caused by mutations that disrupt splicing, but can also be exploited in other ways to address genetic mutations. ${ }^{9}$ ASOs need to be chemically modified to improve stability to nucleases and provide favorable pharmacokinetic and pharmacodynamic characteristics for in vivo use. The most commonly used ASO chemistries are the 2'-O-methyl RNA phosphorothioate chemistry, which is negatively charged, and the charge neutral phosphorodiamidate morpholino oligomer. For more background on ASO chemistry, we refer the reader to a recent book chapter. ${ }^{10}$ This review will describe different ways antisense-mediated splicing modulation can be used to treat genetic diseases. Since there are already numerous reviews describing exon skipping for DMD and SMA, ${ }^{11-14}$ we have chosen to discuss these neuromuscular applications only briefly and to focus on the potential of the exon skipping approach for other genetic diseases. For each approach we have chosen a few illustrative examples, focusing on those where data from animal models is available.

\section{Cryptic splicing}

Cryptic splice sites are sequences in the pre-messenger RNA (pre-mRNA) that are not normally used as splice sites, but which are activated by mutations that either inactivate the canonical splice sites or create splice sites where one did not exist before. ${ }^{15}$ The classical example of this phenomenon is in the $\beta$-globin gene, where mutations in the canonical splice sites cause activation of cryptic splice sites, leading to the inclusion of aberrant exons, resulting in nonfunctional transcripts, defective $\beta$-globin expression, and consequently $\beta$-thalassemia. ${ }^{16}$ For the majority of patients, the disease is caused by mutations that activate cryptic splice sites. ${ }^{17}$ In the early 1990s, ASOs were developed by Dominski and Kole using this paradigm (Figure 1A): by targeting the cryptic splice sites with 2'-O-methyl RNA ASOs to block the access of the spliceosome to these sites, their group was able to restore normal $\beta$-globin splicing in cells ${ }^{18}$ and normal hemoglobin expression, to some extent, in a thalassemia mouse model. ${ }^{19}$ The restoration of normal splicing by suppressing cryptic splicing, therefore, represents the very earliest manifestation of ASOs being used to modulate splicing for therapy. Even despite this early progress, clinical applications of this technology for the treatment of $\beta$-thalassemia have not materialized, likely as a result of the need to generate, validate, and trial specific ASOs for each type of mutation in patients, and the fact that ASO therapy only leads to a transient correction of splicing. As a result, most gene therapy research in the field has concentrated on viral-mediated transduction of $\beta$-globin into hematopoietic stem cells as a means of achieving durable and long-term correction of $\beta$-globin expression, with one patient achieving independence from transfusions, the usual treatment for $\beta$-thalassemia. ${ }^{20}$

Even though in $\beta$-thalassemia, ASO-mediated correction is being side-lined in favor of other approaches, for other diseases targets that could be amenable to ASOmediated cryptic splice site suppression continue to be identified. A recent example is Usher syndrome type 1C, characterized by congenital deafness, retinitis pigmentosa, and vestibular dysfunction (Figure 1B). This is caused by defects in the USHIC gene that encodes harmonin. Harmonin is an actin-binding scaffold protein that, together with myosin VIIa and cadherin 23, are required to ensure that the stereocilia in the inner ear cohere together. ${ }^{21,22}$ The USH1C216G $>$ A mutation, found in all Louisiana Acadian cases of Usher syndrome type $1 \mathrm{C}$, creates a cryptic $5^{\prime}$ splice site within exon 3 of the gene, which is used preferentially over the canonical 5' splice site, and the creation of a frameshift and premature truncation of the harmonin protein. ${ }^{23} \mathrm{In}$ a collaborative project, Lentz et al were able to show that an ASO targeted to the cryptic $5^{\prime}$ splice site was able to correct the splicing of USHIC transcript, restore harmonin expression, and rescue cochlear hair cells, vestibular function, and hearing for at least 6 months when given to an animal model of USH1C216G $>$ A. ${ }^{24}$

\section{Alternative splicing}

In humans, a myriad of proteins is responsible for most of the functions that form the basis of life. However, this array of different proteins is coded by a much (3-4-fold) smaller number of genes. This puzzled the field at first, ${ }^{25}$ but can be explained by the occurrence of alternative splicing, a mechanism that allows the production of several proteins from the same gene by means of the exclusion, inclusion, or trimming of specific exons. In fact, $95 \%$ of human pre-mRNA is alternatively spliced. ${ }^{26}$ Alternative splicing is responsible for generating proteins with very different, sometimes even antagonistic roles. Alternative splicing can also introduce stop codons that cause nonsense-mediated decay, and therefore, suppress the expression of the gene. ${ }^{27}$ In healthy individuals, this flexibility contributes to the clockwork functioning of human biology. However, aberrant alternative splicing can also be the origin of disease, eg, a shift from an antiproliferative isoform to a proproliferative isoform can underlie tumor formation. From the therapeutic viewpoint, this provides researchers 
A
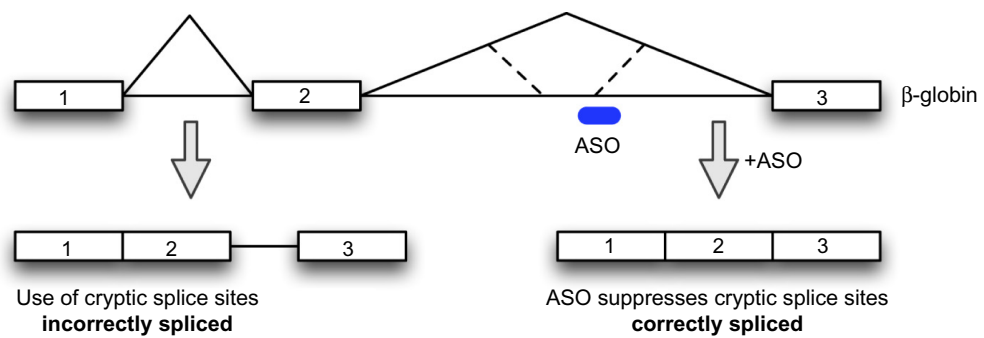

incorrectly spliced correctly spliced

B
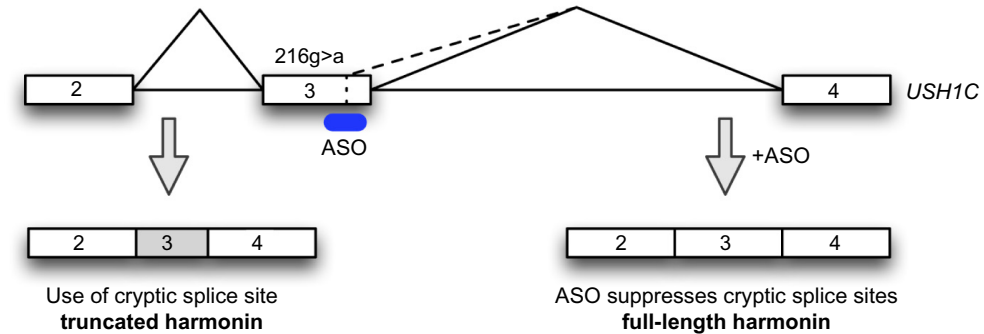

C
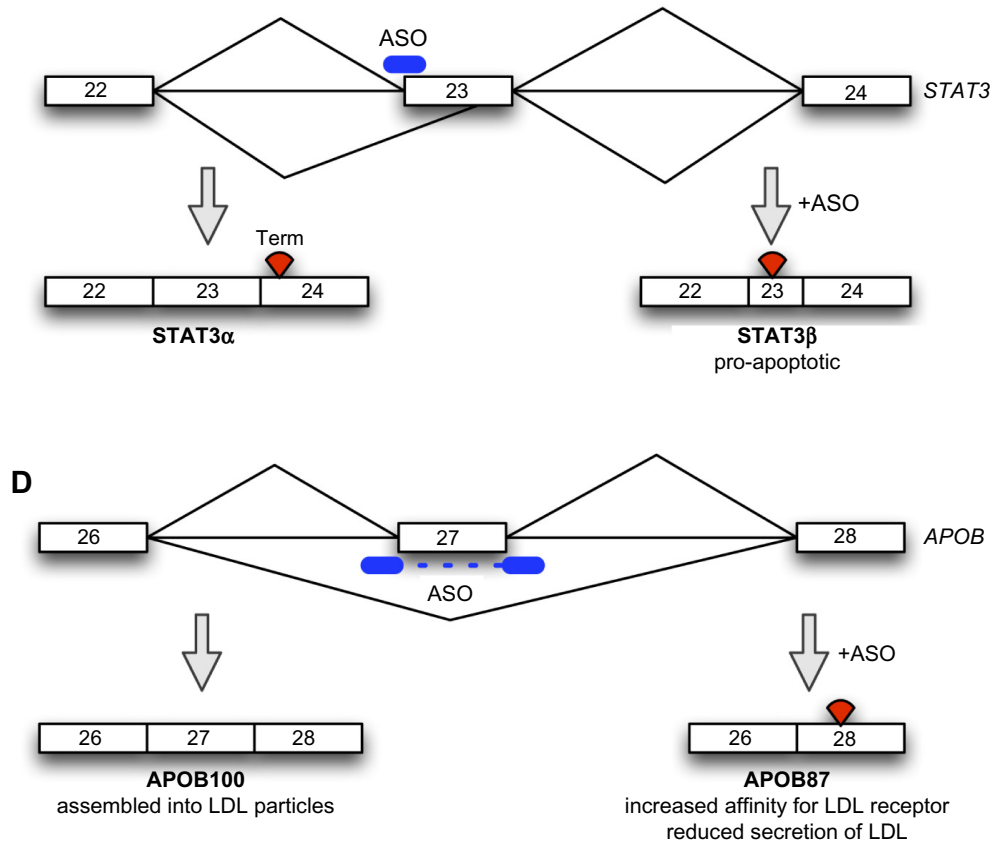

Figure I Schematic depiction of antisense-mediated splicing modulation approaches.

Notes: (A) Restoration of cryptic splicing for $\beta$-globin mutations. Due to a mutation in intron 3, a cryptic exon is included into the $\beta$-globin mRNA, abrogating the production of $\beta$-globin proteins. ASOs targeting the cryptic splice sites prevent the inclusion of the cryptic exon, restoring normal splicing and production of $\beta$-globin protein. (B) Restoration of cryptic splicing for USHIC. Due to a mutation in exon 3, a cryptic splice site within exon 3 is activated, leading to the exclusion of the last part of the exon from the USCHIC transcript and loss of harmonin protein production. Using ASOs targeting the cryptic splice site in exon 3 reactivates the original splice site and restores normal splicing and protein production. (C) Shifting of alternative splicing. The STAT3 gene produces STAT3 $\alpha$ and STAT3 $\beta$ proteins based on the use of a proximal or more distal acceptor splice site in exon 23 during pre-mRNA splicing. ASOs targeting the proximal acceptor splice site lead to a shift into the production of the proapoptotic STAT3 $\beta$ protein. (D) Isoform-specific knockdown. The APOB gene gives rise to APOBI00 and APOB48 proteins. The latter derives from RNA editing in exon 26 . Skipping of exon 27 gives rise to a prematurely truncated APOB protein with cholesterol-lowering properties, without affecting the APOB48 isoform.

Abbreviations: APOB, apolipoprotein BI00; ASOs, antisense oligonucleotides; LDL, low-density lipoprotein; mRNA, messenger RNA; STAT3, signal transducer and activator of transcription 3; USHIC, Usher syndrome type IC gene.

the opportunity to use ASOs as tools to knowingly shift alternative splicing to treat disease.

A first example involves lamin A, a nuclear envelope protein involved in the regulation of gene expression, nuclear stability, and chromatin structure that is encoded by the
$L M N A$ gene. A point mutation in this gene leads to a shift in alternative splicing, ${ }^{28}$ and increased production of progerin, a truncated form of lamin A that is present in small amounts in normal aging, but which accumulates in high levels in HGPS. This leads to accelerated aging and shortened life 
span due to growth impairment, lipodystrophy, dermal and bone abnormalities, and cardiovascular alterations. ${ }^{29}$ This shift in the standard splicing of the $L M N A$ gene favoring the truncated progerin protein represents an ideal example of how the usually firmly kept balance can be disturbed by the presence of this cryptic splice site: the new protein is only 50 amino acids shorter than the original one, but lacks a vital target sequence for protein cleavage, ${ }^{30}$ and this in turn leads to the deleterious effects experienced by HGPS patients. A phosphorodiamidate morpholino oligomer ASO masking the progerin splice site has been successfully used in fibroblast cultures from HGPS patients to restore normal splicing and correct nuclear abnormalities and mislocalization of other nuclear envelope proteins. ${ }^{31}$ Following these advances, in a recent study on a murine model that recapitulates the main clinical signs of HGPS, vivo-morpholino ASOs were able to reverse many of the phenotypical alterations seen in these mice. ${ }^{32}$

Signal transducer and activator of transcription 3 (STAT3) is involved in the activation of several oncogenic pathways. It is usually spliced to the isoform STAT3 $\alpha$, but an alternative acceptor site in exon 23 leads to the expression of isoform STAT3 $\beta$, which lacks the C-terminal transactivation domain. Overexpression of STAT $\beta$ can induce apoptosis and inhibit tumor growth. Zammarchi et al developed an ASO targeting a splicing enhancer site that was able to shift the balance of STAT3 expression to favor STAT3 $\beta$ expression (Figure 1C). ${ }^{33}$ This approach has been tested in cell and animal models and has served a double purpose: on one hand it allowed a detailed study of the precise functions of STAT3 $\beta$ in its physiological environment. On the other hand, ASOs that shift splicing to the STAT3 $\beta$ isoform have antitumor applications.

\section{Knockdown}

Exon-skipping ASOs can also be used to knockdown the function of an undesired gene, by inducing exon skipping and creating mRNA isoforms that encode nonfunctional proteins or trigger nonsense-mediated decay of the mRNA, ${ }^{34}$ to inhibit the expression of the undesired gene; or encode alternative isoforms with desired physiological or therapeutic functions that could modify or antagonize the effect of an undesired gene. The use of exon-skipping ASOs for the first purpose is not generally widespread except in the special case of zebrafish genetics. ${ }^{35}$ Other methods, such as RNA interference, ${ }^{36}$ ASOs that trigger RNase H-mediated degradation of mRNAs, ${ }^{37}$ or translation inhibition, ${ }^{38}$ are more generally used if simple knockdown is desired. An example of this is the knockdown of apolipoprotein B100 (APOB100) as a means of reducing low-density lipoprotein (LDL) secretion and cholesterol levels in familial hypercholesterolemia. ${ }^{39}$ Both RNase H "gap-mer" ASOs and RNA interference have been used for this purpose, reducing LDL cholesterol in vivo. ${ }^{40,41}$ These simple knockdown approaches, however, have the undesired effect of knocking down the APOB48 isoform, and therefore reduce chylomicron secretion. ${ }^{42}$ This will interfere with fat transport from the intestine and malabsorption of fat-soluble vitamins such as vitamin K. ${ }^{43}$

Exon-skipping ASOs offer a distinct advantage over simple knockdown, as engineered alternative isoforms may possess their own therapeutic properties, inhibit the function of a normal isoform, and/or avoid side effects of knockdown of the normal isoform. Capitalizing on this advantage, exonskipping exon 27 of the $A P O B$ gene using ASOs causes a reduction of LDL particle secretion and an increase in LDL affinity for the LDL receptor (Figure 1D). ${ }^{44}$ These two effects combine to cause a powerful reduction of LDL cholesterol when the "APO-skip" ASOs are introduced into hypercholesterolemic mice transgenic for human $A P O B$, validating the therapeutic concept. ${ }^{45}$ Importantly, exon 27 skipping does not affect APOB48 expression, as the RNA editing necessary for APOB48 expression occurs in exon $26 .{ }^{45}$ In this example, therefore, exon-skipping technology enables the reengineering of gene expression to produce a desired outcome with clear therapeutic advantages over other approaches to the same problem.

\section{Reading frame correction}

The coding sequence for proteins is dispersed over exons, and the location where introns interrupt the code can be in three phases in relation to the coding sequence: either between complete codons (phase 0 introns), interrupting after the first nucleotide of the codon (phase 1 introns), or interrupting after the second nucleotide of the codon (phase 2 introns). As this will apply to both the beginning and the end of each exon, each exon will have two jigsaw-like ends that will require a complementary exon before and after to maintain the open reading frame $(\mathrm{ORF}) .{ }^{46}$ Therefore, a symmetrical exon can be deleted without interrupting the ORF, but others, if deleted, would cause a disruption in the ORF (Figure 2). For most genes, the deletion of an exon would result in a nonfunctional protein regardless of whether the ORF is disrupted by the deletion, since the majority of proteins do not have redundant domains. However, there are some exceptions where deletions that maintain the ORF allow the production of internally deleted but partially functional proteins. This concept is best 
A

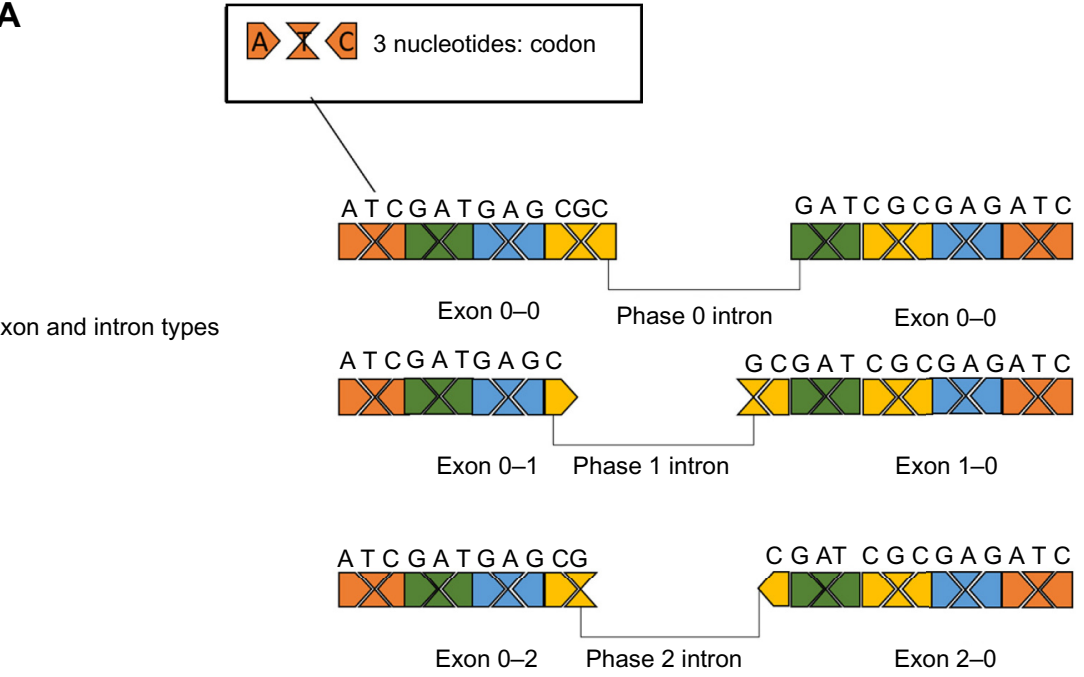

B

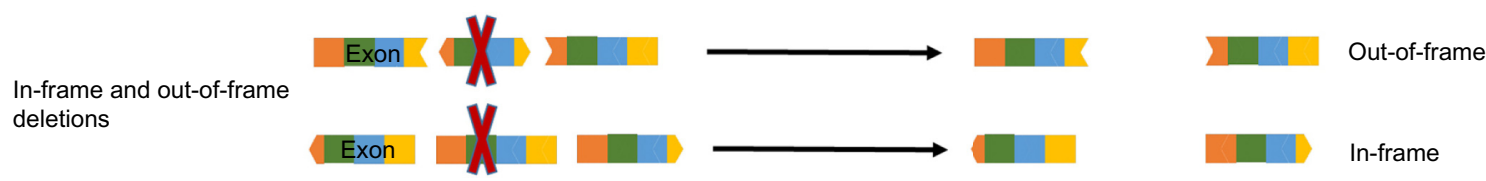

Figure 2 Effect of exon deletions on the open reading frame.

Notes: (A) Different ways in which codons in exons may be split by introns: in phase 0 introns, the codon ends at the end of the exon, while in phase I and phase 2 introns the codon is split over the 2 exons, with the interruption after the first or the second nucleotide of the codon, respectively. (B) As each exon is separated from the next and previous one by the flanking introns, there are nine different types of exons due to the different combinations $(0-0,0-1,0-2, I-0, I-I, I-2,2-0,2-I, 2-2)$; if one of these exons is deleted, the open reading frame could be either disrupted or maintained, depending on these combinations: the deletion of "symmetrical" exons ( $0-0$, I-I, 2-2) would have no effect on the reading frame (in-frame), while others will disrupt it (out-of-frame).

illustrated in the neuromuscular disorder DMD, an early onset, severely progressive muscle-wasting disorder. ${ }^{47}$ In the majority of DMD patients, deletions or duplications of one or more exons cause a disruption of the ORF and the deficit of its encoded protein, dystrophin. ${ }^{48}$ In a stark contrast to DMD, another disorder in which one or more exons are also deleted from the $D M D$ gene, Becker muscular dystrophy, has a much milder progression. The difference is that in Becker patients, the deletions do not disrupt the ORF and a shorter, but partially functional dystrophin protein is produced ${ }^{48}$ The existence of Becker muscular dystrophy is the conceptual basis for the model of exon skipping: ASOs are used to "mask" specific exons to the splicing machinery to restore the ORF and partial dystrophin function for DMD patients.

From early proof of concept experiments to the latest clinical trials, the developments in the use of ASOs as a potential therapeutic agent in DMD have been fast: restoration of dystrophin has been achieved following intramuscular ${ }^{49,50}$ and systemic ${ }^{4,5,51}$ injections of ASOs targeting DMD exon 51, and these and several other ASOs targeting other exons are progressing through clinical trials. ${ }^{52}$

SMA, the most common genetic cause of death in infants under the age of 2 years, is caused by deletions and mutations of SMN1, and consequently low levels of SMN protein, a ubiquitously expressed protein involved in RNA processing. ${ }^{53}$ Although a duplicate of SMN1, SMN2 also exists within the genome and also encodes SMN; it is usually inefficiently spliced due to minor differences in and around exon 7 . As a result, only $20 \%$ of SMN2 mRNA transcripts include exon 7, which is necessary for the stability of SMN protein. ${ }^{54}$ In healthy individuals, this is irrelevant, but in SMA patients, increasing the amount of exon 7 inclusion from SMN2 transcripts could allow the generation of sufficient amounts of SMN protein to ameliorate their disease. In fact, several studies using ASOs have already demonstrated in vitro and in vivo that this is possible, ${ }^{6,55-58}$ and clinical trials are underway.

\section{Toward clinical application}

From the above, it is clear that antisense-mediated splicing modulation may offer a potential treatment for rare inherited diseases, and as such, can be seen as a platform approach. For DMD and SMA, the approach has made it to the clinical trial phase, whereas other genetic diseases are still in preclinical development. While each of these diseases will have their own quirks and challenges (eg, delivery to the tar- 
get tissue and dosing regimens will vary between diseases), there will be common challenges and hurdles as well, since they all use the exon-skipping platform. Furthermore, there is no reason why people embarking on developing exon skipping for their disease of choice should have to repeat mistakes others have made in the past. Therefore, networking between scientists involved in this endeavor is warranted. The European Cooperation of Science and Technology (COST) provides funding for networking to overcome challenges such as these, and has funded the Action "Networking towards clinical application of antisense-mediated exon skipping for rare diseases" (BM1207, see http://www.cost.eu and http://www.exonskipping.eu). ${ }^{59}$ Within this Action, regular workshops are organized to discuss common challenges, such as delivery to the target tissue, ASO chemistry and safety, and to align preclinical work. Furthermore, the Action focuses on the development of new regulatory models and has recently had a workshop on translational and regulatory challenges with representatives from patient organizations, academia, and regulatory agencies, ${ }^{60}$ following a larger workshop organized by the Translational Research in Europe - Assessment and Treatment of Neuromuscular Diseases (TREAT-NMD) network and hosted by the European Medicines Agency that focused on the particulars of exon skipping for DMD. ${ }^{61}$

One of the key factors for clinical development will be the harmonization of outcome measures. While the regulators will provide market approval only when clinically relevant benefit has been convincingly shown, biochemical outcome measures can provide additional information, eg, by confirming mechanism of action of ASOs. Lessons could be learned from the case of DMD: when the first clinical trials using ASOs were outlined, methods to measure dystrophin restoration relied heavily on standard clinical diagnostic techniques and academic protocols, and each research center used their own version of the methods. , $5,49,51,52^{\text {The most }}$ commonly used method was immunofluorescent staining, but the large size and low expression level of the protein made accurate quantification technically challenging, especially when the amount of restored dystrophin is relatively small and it needs to be distinguished from preexisting dystrophinpositive revertant fibers and residual trace dystrophin expression. ${ }^{52,62}$ To overcome some of those difficulties, refinements developed, in particular using better software analysis. ${ }^{63,64}$ A group of laboratories from academia and industry have embarked on an effort to provide a data-driven reproducible methodology for dystrophin quantification that could be used in future trials. This group has revised methodologies, validated them among their laboratories, and proposed a standard operating procedure..$^{52,65}$

\section{Conclusion}

ASO-mediated splicing modulation holds potential for treating rare genetic diseases as outlined in this review, and as corroborated by the advances made for DMD and SMA. International networks on exon skipping aim to help scientists developing this approach for other diseases benefit from the experience gained from the DMD field, to jointly overcome challenges and further this approach toward clinical application for as many rare genetic diseases as possible.

\section{Acknowledgments}

Authors gratefully acknowledge funding of the COST Action BM1207 by the Cooperation of Science and Technology (COST). Virginia Arechavala-Gomeza is funded by a Miguel Servet Fellowship (Spanish Health Institute Carlos III) and a Marie Curie Career Integration Grant (European Commission).

\section{Disclosure}

Dr Khoo discloses being co-inventor on UK Patent GB1117880.3. Dr Aartsma-Rus discloses being employed by the Leiden University Medical Center (LUMC) which has patents on exon skipping. Dr Aartsma-Rus is co-inventor on some of these patents and as such is entitled to a share of potential royalties. Dr Arechavala-Gomeza has nothing to declare.

\section{References}

1. Kaplan JC, Hamroun D. The 2014 version of the gene table of monogenic neuromuscular disorders (nuclear genome). Neuromuscul Disord. 2013;23(12):1081-1111

2. Bertrand AT, Chikhaoui K, Yaou RB, Bonne G. Clinical and genetic heterogeneity in laminopathies. Biochem Soc Trans. 2011;39(6): $1687-1692$.

3. Lewandowska MA. The missing puzzle piece: splicing mutations. Int $J$ Clin Exp Pathol. 2013;6(12):2675-2682.

4. Cirak S, Arechavala-Gomeza V, Guglieri M, et al. Exon skipping and dystrophin restoration in patients with Duchenne muscular dystrophy after systemic phosphorodiamidate morpholino oligomer treatment: an open-label, phase 2, dose-escalation study. Lancet. 2011; 378(9791):595-605.

5. Goemans NM, Tulinius M, van den Akker JT, et al. Systemic administration of PRO051 in Duchenne's muscular dystrophy. $N$ Engl J Med. 2011;364(16):1513-1522.

6. Hua Y, Sahashi K, Hung G, et al. Antisense correction of SMN2 splicing in the CNS rescues necrosis in a type III SMA mouse model. Genes Dev. 2010;24(15):1634-1644.

7. Zanetta C, Nizzardo M, Simone C, et al. Molecular therapeutic strategies for spinal muscular atrophies: current and future clinical trials. Clin Ther. 2014;36(1):128-140. 
8. Yokota T, Nakamura A, Nagata T, et al. Extensive and prolonged restoration of dystrophin expression with vivo-morpholino-mediated multiple exon skipping in dystrophic dogs. Nucleic Acid Ther. 2012;22(5): 306-315.

9. van Roon-Mom WM, Aartsma-Rus A. Overview on applications of antisense-mediated exon skipping. Methods Mol Biol. 2012;867: 79-96.

10. Saleh AF, Arzumanov AA, Gait MJ. Overview of alternative oligonucleotide chemistries for exon skipping. Methods Mol Biol 2012;867:365-378.

11. Douglas AG, Wood MJ. Splicing therapy for neuromuscular disease. Mol Cell Neurosci. 2013;56:169-185.

12. Foster H, Popplewell L, Dickson G. Genetic therapeutic approaches for Duchenne muscular dystrophy. Hum Gene Ther. 2012;23(7): 676-687.

13. Aartsma-Rus A. Antisense-mediated modulation of splicing: therapeutic implications for Duchenne muscular dystrophy. RNA Biol. 2010;7(4):453-461.

14. Arechavala-Gomeza V, Anthony K, Morgan J, Muntoni F. Antisense oligonucleotide-mediated exon skipping for Duchenne muscular dystrophy: progress and challenges. Curr Gene Ther. 2012;12(3): 152-160.

15. Roca X, Krainer AR, Eperon IC. Pick one, but be quick: $5^{\prime}$ splice sites and the problems of too many choices. Genes Dev. 2013;27(2):129-144.

16. Wieringa B, Meyer F, Reiser J, Weissmann C. Unusual splice sites revealed by mutagenic inactivation of an authentic splice site of the rabbit beta-globin gene. Nature. 1983;301(5895):38-43.

17. Busslinger M, Moschonas N, Flavell RA. Beta + thalassemia: aberrant splicing results from a single point mutation in an intron. Cell. 1981 27(2 Pt 1):289-298.

18. Dominski Z, Kole R. Restoration of correct splicing in thalassemic pre-mRNA by antisense oligonucleotides. Proc Natl Acad Sci U S A. 1993;90(18):8673-8677.

19. Svasti S, Suwanmanee T, Fucharoen S, et al. RNA repair restores hemoglobin expression in IVS2-654 thalassemic mice. Proc Natl Acad Sci U S A. 2009;106(4):1205-1210.

20. Cavazzana-Calvo M, Payen E, Negre O, et al. Transfusion independence and HMGA2 activation after gene therapy of human beta-thalassaemia. Nature. 2010;467(7313):318-322.

21. Boeda B, El-Amraoui A, Bahloul A, et al. Myosin VIIa, harmonin and cadherin 23, three Usher I gene products that cooperate to shape the sensory hair cell bundle. EMBO J. 2002;21(24):6689-6699.

22. Bahloul A, Michel V, Hardelin JP, et al. Cadherin-23, myosin VIIa and harmonin, encoded by Usher syndrome type I genes, form a ternary complex and interact with membrane phospholipids. Hum Mol Genet. 2010;19(18):3557-3565.

23. Lentz J, Savas S, Ng SS, Athas G, Deininger P, Keats B. The USH1C $216 \mathrm{G}-->$ A splice-site mutation results in a 35-base-pair deletion. Hum Genet. 2005;116(3):225-227.

24. Lentz JJ, Jodelka FM, Hinrich AJ, et al. Rescue of hearing and vestibular function by antisense oligonucleotides in a mouse model of human deafness. Nat Med. 2013;19(3):345-350.

25. Pennisi E. Why do humans have so few genes? Science. 2005; 309(5731):80.

26. Johnson JM, Castle J, Garrett-Engele P, et al. Genome-wide survey of human alternative pre-mRNA splicing with exon junction microarrays. Science. 2003;302(5653):2141-2144.

27. Stoilov P, Daoud R, Nayler O, Stamm S. Human tra2-beta1 autoregulates its protein concentration by influencing alternative splicing of its pre-mRNA. Hum Mol Genet. 2004;13(5):509-524.

28. Eriksson M, Brown WT, Gordon LB, et al. Recurrent de novo point mutations in lamin A cause Hutchinson-Gilford progeria syndrome. Nature. 2003;423(6937):293-298.

29. Hennekam RC. Hutchinson-Gilford progeria syndrome: review of the phenotype. Am J Med Genet A. 2006;140(23):2603-2624

30. Gruenbaum Y, Margalit A, Goldman RD, Shumaker DK, Wilson KL. The nuclear lamina comes of age. Nat Rev Mol Cell Biol. 2005;6(1): 21-31.
31. Scaffidi P, Misteli T. Reversal of the cellular phenotype in the premature aging disease Hutchinson-Gilford progeria syndrome. Nat Med. 2005;11(4):440-445.

32. Osorio FG, Navarro CL, Cadinanos J, et al. Splicing-directed therapy in a new mouse model of human accelerated aging. Sci Transl Med. 2011;3(106):106ra107.

33. Zammarchi F, de SE, Bournazou E, et al. Antitumorigenic potential of STAT3 alternative splicing modulation. Proc Natl Acad Sci U S A. 2011;108(43):17779-17784.

34. Maquat LE. Nonsense-mediated mRNA decay: splicing, translation and mRNP dynamics. Nat Rev Mol Cell Biol. 2004;5(2):89-99.

35. Draper BW, Morcos PA, Kimmel CB. Inhibition of zebrafish fgf8 pre-mRNA splicing with morpholino oligos: a quantifiable method for gene knockdown. Genesis. 2001;30(3):154-156.

36. McManus MT, Sharp PA. Gene silencing in mammals by small interfering RNAs. Nat Rev Genet. 2002;3(10):737-747.

37. Grunweller A, Wyszko E, Bieber B, Jahnel R, Erdmann VA, Kurreck J. Comparison of different antisense strategies in mammalian cells using locked nucleic acids, 2'-O-methyl RNA, phosphorothioates and small interfering RNA. Nucleic Acids Res. 2003;31(12):3185-3193.

38. McCaffrey AP, Meuse L, Karimi M, Contag CH, Kay MA. A potent and specific morpholino antisense inhibitor of hepatitis $\mathrm{C}$ translation in mice. Hepatology. 2003;38(2):503-508.

39. Marks D, Thorogood M, Neil HA, Humphries SE. A review on the diagnosis, natural history, and treatment of familial hypercholesterolaemia. Atherosclerosis. 2003;168(1):1-14.

40. Zimmermann TS, Lee AC, Akinc A, et al. RNAi-mediated gene silencing in non-human primates. Nature. 2006;441(7089):111-114.

41. Raal FJ, Santos RD, Blom DJ, et al. Mipomersen, an apolipoprotein B synthesis inhibitor, for lowering of LDL cholesterol concentrations in patients with homozygous familial hypercholesterolaemia: a randomised, double-blind, placebo-controlled trial. Lancet. 2010;375(9719):998-1006.

42. Soutschek J, Akinc A, Bramlage B, et al. Therapeutic silencing of an endogenous gene by systemic administration of modified siRNAs. Nature. 2004;432(7014):173-178.

43. Cuchel M, Meagher EA, du Toit TH, et al. Efficacy and safety of a microsomal triglyceride transfer protein inhibitor in patients with homozygous familial hypercholesterolaemia: a single-arm, open-label, phase 3 study. Lancet. 2013;381(9860):40-46.

44. Khoo B, Roca X, Chew SL, Krainer AR. Antisense oligonucleotideinduced alternative splicing of the APOB mRNA generates a novel isoform of APOB. BMC Mol Biol. 2007;8:3.

45. Disterer P, Al-Shawi R, Ellmerich S, et al. Exon skipping of hepatic APOB pre-mRNA with splice-switching oligonucleotides reduces LDL cholesterol in vivo. Mol Ther. 2013;21(3):602-609.

46. Kolkman JA, Stemmer WP. Directed evolution of proteins by exon shuffling. Nat Biotechnol. 2001;19(5):423-428.

47. Eagle M, Baudouin SV, Chandler C, Giddings DR, Bullock R, Bushby K. Survival in Duchenne muscular dystrophy: improvements in life expectancy since 1967 and the impact of home nocturnal ventilation. Neuromuscul Disord. 2002;12(10):926-929.

48. Muntoni F, Torelli S, Ferlini A. Dystrophin and mutations: one gene, several proteins, multiple phenotypes. Lancet Neurol. 2003;2(12): 731-740.

49. Kinali M, Arechavala-Gomeza V, Feng L, et al. Local restoration of dystrophin expression with the morpholino oligomer AVI-4658 in Duchenne muscular dystrophy: a single-blind, placebo-controlled, dose-escalation, proof-of-concept study. Lancet Neurol. 2009;8(10):918-928.

50. van Deutekom JC, Janson AA, Ginjaar IB, et al. Local dystrophin restoration with antisense oligonucleotide PRO051. $N$ Engl J Med. 2007;357(26):2677-2686.

51. Mendell JR, Rodino-Klapac LR, Sahenk Z, et al. Eteplirsen for the treatment of Duchenne muscular dystrophy. Ann Neurol. 2013;74(5): 637-647.

52. Aartsma-Rus A. Dystrophin analysis in clinical trials. J Neuromuscul Dis. 2014;1:41-53. 
53. Lefebvre S, Burglen L, Reboullet S, et al. Identification and characterization of a spinal muscular atrophy-determining gene. Cell. 1995;80(1):155-165.

54. Monani UR, Lorson CL, Parsons DW, et al. A single nucleotide difference that alters splicing patterns distinguishes the SMA gene SMN1 from the copy gene SMN2. Hum Mol Genet. 1999;8(7): 1177-1183.

55. Rigo F, Chun SJ, Norris DA, et al. Pharmacology of a central nervous system delivered 2'-o-methoxyethyl-modified survival of motor neuron splicing oligonucleotide in mice and nonhuman primates. J Pharmacol Exp Ther. 2014;350(1):46-55.

56. Passini MA, Bu J, Richards AM, et al. Antisense oligonucleotides delivered to the mouse CNS ameliorate symptoms of severe spinal muscular atrophy. Sci Transl Med. 2011;3(72):72ra18.

57. Hua Y, Vickers TA, Okunola HL, Bennett CF, Krainer AR. Antisense masking of an hnRNP A1/A2 intronic splicing silencer corrects SMN2 splicing in transgenic mice. Am J Hum Genet. 2008;82(4):834-848.

58. Hua Y, Vickers TA, Baker BF, Bennett CF, Krainer AR. Enhancement of SMN2 exon 7 inclusion by antisense oligonucleotides targeting the exon. PLoS Biol. 2007;5(4):e73.

59. Aartsma-Rus A. Antisense-mediated exon skipping: networking to meet opportunities and to overcome challenges. Nucleic Acid Ther. 2014;24(1):1-3.
60. Aartsma-Rus A, Ferlini A, Goemans N, et al. Translational and regulatory challenges for exon skipping therapies. Hum Gene Ther. 2014;25(10):885-892.

61. Muntoni F; Meeting Steering Committee and TREAT-NMD Network. The development of antisense oligonucleotide therapies for Duchenne muscular dystrophy: report on a TREAT-NMD workshop hosted by the European Medicines Agency (EMA), on September 25th 2009. Neuromuscul Disord. 2010;20(5):355-362.

62. Arechavala-Gomeza V, Kinali M, Feng L, et al. Revertant fibres and dystrophin traces in Duchenne muscular dystrophy: implication for clinical trials. Neuromuscul Disord. 2010;20(5):295-301.

63. Arechavala-Gomeza V, Kinali M, Feng L, et al. Immunohistological intensity measurements as a tool to assess sarcolemma-associated protein expression. Neuropathol Appl Neurobiol. 2010;36(4):265-274.

64. Taylor LE, Kaminoh YJ, Rodesch CK, Flanigan KM. Quantification of dystrophin immunofluorescence in dystrophinopathy muscle specimens. Neuropathol Appl Neurobiol. 2012;38(6):591-601.

65. Anthony K, Arechavala-Gomeza V, Taylor LE, et al. Dystrophin quantification: Biological and translational researcher implications. Neurology. Epub 2014 October 29.
The Application of Clinical Genetics

\section{Publish your work in this journal}

The Application of Clinical Genetics is an international, peer-reviewed open access journal that welcomes laboratory and clinical findings in the field of human genetics. Specific topics include: Population genetics; Functional genetics; Natural history of genetic disease; Management of genetic disease; Mechanisms of genetic disease; Counseling and ethical

\section{Dovepress}

issues; Animal models; Pharmacogenetics; Prenatal diagnosis; Dysmorphology. The manuscript management system is completely online and includes a very quick and fair peer-review system, which is all easy to use. Visit http://www.dovepress.com/testimonials.php to read real quotes from published authors. 